UMTG-312

\title{
Preparing exact eigenstates of the open XXZ chain on a quantum computer
}

\author{
John S. Van Dyke® Edwin Barnes ${ }^{1}$, Sophia E. Economou ${ }^{1}$, \\ and Rafael I. Nepomechie $\mathrm{L}^{2}$
}

\begin{abstract}
The open spin-1/2 XXZ spin chain with diagonal boundary magnetic fields is the paradigmatic example of a quantum integrable model with open boundary conditions. We formulate a quantum algorithm for preparing Bethe states of this model, corresponding to real solutions of the Bethe equations. The algorithm is probabilistic, with a success probability that decreases with the number of down spins. For a Bethe state of $L$ spins with $M$ down spins, which contains a total of $\left(\begin{array}{l}L \\ M\end{array}\right) 2^{M} M$ ! terms, the algorithm requires $L+M^{2}+2 M$ qubits.
\end{abstract}

\footnotetext{
${ }^{1}$ Department of Physics, Virginia Tech, Blacksburg, VA 24061 USA

${ }^{2}$ Physics Department, P.O. Box 248046, University of Miami, Coral Gables, FL 33124 USA

efbarnes@vt.edu, economou@vt.edu, nepomechie@miami.edu, jvandyke@vt.edu
} 


\section{Introduction}

The existence of exactly solvable (or quantum integrable) interacting many-body quantum models, the first example of which appeared already 90 years ago [1], is remarkable. There are infinitely many such models, since they originate [2] from solutions of the Yang-Baxter equation [3], of which there are infinitely many. These models have had, and continue to have, a significant impact in theoretical physics, ranging from condensed matter physics and statistical mechanics to string theory [4].

Although much is already known about quantum integrable models, there is much that remains unknown. This is due largely to the fact their exact solutions depend on solutions of corresponding Bethe equations; and the latter are generally hard to solve. Hence, despite the fact that the models are "exactly solvable," significant effort is generally still necessary to explicitly compute quantities of physical interest.

Quantum computers hold the promise of addressing a variety of heretofore intractable problems [5, 6]. These include quantum simulation of many-body systems, which occur in molecular and solid-state contexts [7, 8]. It is natural to ask whether quantum computers could also help address the problem of computing quantities of physical interest for quantum integrable models. While solving Bethe equations remains an interesting open challenge [9], an important recent development is the discovery of an efficient quantum algorithm for constructing exact eigenstates [10]. This algorithm could potentially be used for explicitly computing correlation functions, which would otherwise be out of reach.

Integrable models could also impact quantum computing by providing a testbed for quantum simulators. While there is a large ongoing effort to develop near-term algorithms, such as variational quantum eigensolvers (VQEs) [11, 12], to solve many-body problems, it is not clear whether a quantum advantage can be achieved by VQEs on near-term hardware. On the other hand, obtaining quantum advantage for generic simulation problems on fault-tolerant quantum computers is believed to be enormously costly in terms of quantum resources 13 15. An additional benefit of integrable models for early quantum computers beyond the noisy intermediate-scale quantum (NISQ) era [16 is that their classically solvable quantities can be used for validation and verification purposes. It is therefore natural to investigate special classes of problems, such as integrable models, for earlier demonstrations of quantum advantage. A key first step is to find quantum algorithms that solve such problems and to quantify the required resources.

The algorithm in [10] is for the closed spin-1/2 XXZ spin chain, which is an anisotropic version [17] of the model solved by Bethe [1], and which is the paradigmatic example of a quantum integrable model with periodic boundary conditions. The extension of quantum

integrability to models with open boundary conditions is also interesting and nontrivial, see e.g. 18 21 and related references.

In this paper we formulate a quantum algorithm for constructing exact eigenstates of the open spin-1/2 XXZ spin chain with diagonal boundary magnetic fields, which is the paradigmatic example of a quantum integrable model with open boundary conditions. The 
(ferromagnetic) Hamiltonian $\mathcal{H}$ for a chain with length $L$ is given by

$$
\mathcal{H}=-\frac{1}{2} \sum_{n=0}^{L-2}\left(\sigma_{n}^{x} \sigma_{n+1}^{x}+\sigma_{n}^{y} \sigma_{n+1}^{y}+\Delta \sigma_{n}^{z} \sigma_{n+1}^{z}\right)-\frac{1}{2}\left(h \sigma_{0}^{z}+h^{\prime} \sigma_{L-1}^{z}\right)
$$

where as usual $\sigma_{n}^{x}, \sigma_{n}^{y}, \sigma_{n}^{z}$ are Pauli matrices at site $n$. The Hamiltonian has three parameters: the anisotropy parameter $\Delta$, and the boundary magnetic fields $h$ and $h^{\prime}$, all of which are assumed here to be real.

An interesting feature of this model is that, for the special values of the boundary magnetic fields

$$
h^{\prime}=-h=\frac{1}{2}\left(q-q^{-1}\right), \quad \text { where } \quad \Delta=\frac{1}{2}\left(q+q^{-1}\right),
$$

the model has the quantum group symmetry $U_{q}(s u(2))[22,23]$. Consequently, the spectrum has the degeneracies of an isotropic (su(2)-invariant) model, even if $\Delta \neq 1$.

While there are many similarities between the closed-chain and open-chain algorithms, the latter has some new features due to the greater complexity of open-chain Bethe states. As in [10], we restrict here to solutions of the Bethe equations that are real, which can be efficiently determined classically, and which serve as inputs for the quantum algorithm. The quantum algorithm has as outputs exact eigenstates of the Hamiltonian (1.1), with a probability that decreases with the number of down spins. We expect that these eigenstates could be used to explicitly compute the model's correlation functions, beyond what can be done analytically [24, 25].

The outline of the remainder of this paper is as follows. In Sec. 2, we briefly review the model's coordinate Bethe ansatz solution [19], and we recast it in a form that is convenient for our algorithm. We present the algorithm in Sec. 3. In Sec. 4, we summarize the results of our simulations of this algorithm. We conclude in Sec. 5 with a discussion of our results. The complete circuit for the simple case $L=4, M=2$ is provided in Appendix $\mathrm{A}$.

\section{Coordinate Bethe ansatz}

We briefly recall here the coordinate Bethe ansatz solution [19] of the model, and recast it in a form that is convenient for our algorithm, see (2.20)-(2.22) below. (The solution for the case without boundary magnetic fields was found in [18], and the algebraic Bethe ansatz solution was formulated in $[20]$.) The Bethe states are exact eigenstates $|M\rangle$ of the Hamiltonian (1.1)

$$
\mathcal{H}|M\rangle=E|M\rangle
$$

which are given by

$$
|M\rangle=\sum_{0 \leq x_{0}<x_{1}<\ldots<x_{M-1} \leq L-1} f\left(x_{0}, \ldots, x_{M-1}\right)\left|x_{0}, \ldots, x_{M-1}\right\rangle,
$$


where $x_{0}, \ldots, x_{M-1}$ denote the location of the $M$ down spins. In other words,

$$
\left|x_{0}, \ldots, x_{M-1}\right\rangle=\sigma_{x_{0}}^{-} \ldots \sigma_{x_{M-1}}^{-}|0 \ldots 0\rangle
$$

where $\sigma_{n}^{-}=\frac{1}{2}\left(\sigma_{n}^{x}-i \sigma_{n}^{y}\right)$ is the spin-lowering operator at site $n$, and $|0 \ldots 0\rangle$ is the ferromagnetic ground state (i.e., the reference state with all $L$ spins in the up-state $|0\rangle=\left(\begin{array}{l}1 \\ 0\end{array}\right)$.) Moreover, the wave function $f\left(x_{0}, \ldots, x_{M-1}\right)$ is given by

$$
f\left(x_{0}, \ldots, x_{M-1}\right)=\sum_{P} \varepsilon_{P} A\left(k_{0}, \ldots, k_{M-1}\right) e^{i \sum_{j=0}^{M-1} k_{j} x_{j}},
$$

where the sum is over all permutations and negations of $k_{0}, \ldots, k_{M-1}$, and $\varepsilon_{P}= \pm 1$ changes sign at each such mutation. For example, for $M=2$, the wave function is given by

$$
\begin{aligned}
f\left(x_{0}, x_{1}\right) & =A\left(k_{0}, k_{1}\right) e^{i\left(k_{0} x_{0}+k_{1} x_{1}\right)}-A\left(-k_{0}, k_{1}\right) e^{i\left(-k_{0} x_{0}+k_{1} x_{1}\right)} \\
& -A\left(k_{0},-k_{1}\right) e^{i\left(k_{0} x_{0}-k_{1} x_{1}\right)}+A\left(-k_{0},-k_{1}\right) e^{i\left(-k_{0} x_{0}-k_{1} x_{1}\right)} \\
& -A\left(k_{1}, k_{0}\right) e^{i\left(k_{1} x_{0}+k_{0} x_{1}\right)}+A\left(-k_{1}, k_{0}\right) e^{i\left(-k_{1} x_{0}+k_{0} x_{1}\right)} \\
& +A\left(k_{1},-k_{0}\right) e^{i\left(k_{1} x_{0}-k_{0} x_{1}\right)}-A\left(-k_{1},-k_{0}\right) e^{i\left(-k_{1} x_{0}-k_{0} x_{1}\right)}
\end{aligned}
$$

In general, there are $2^{M} M$ ! terms for fixed $x_{0}, \ldots, x_{M-1}$, while the wave function for the corresponding closed chain has only $M$ ! terms, since in the latter case there is a sum only over the permutations.

The coefficients $A\left(k_{0}, \ldots, k_{M-1}\right)$ are given by

$$
A\left(k_{0}, \ldots, k_{M-1}\right)=\prod_{j=0}^{M-1} \beta\left(-k_{j}\right) \prod_{0 \leq j<l \leq M-1} B\left(-k_{j}, k_{l}\right) e^{-i k_{l}}
$$

where

$$
B\left(k, k^{\prime}\right)=s\left(k, k^{\prime}\right) s\left(k^{\prime}, k\right), \quad s\left(k, k^{\prime}\right)=1-2 \Delta e^{i k^{\prime}}+e^{i\left(k+k^{\prime}\right)} .
$$

The so-called Bethe roots $k_{0}, \ldots, k_{M-1}$ satisfy the Bethe equations

$$
\frac{\alpha\left(k_{j}\right) \beta\left(k_{j}\right)}{\alpha\left(-k_{j}\right) \beta\left(-k_{j}\right)}=\prod_{l=0 ; l \neq j}^{M-1} \frac{B\left(-k_{j}, k_{l}\right)}{B\left(k_{j}, k_{l}\right)}, \quad j=0, \ldots, M-1
$$

where

$$
\begin{aligned}
& \alpha(k)=1+(h-\Delta) e^{-i k}, \\
& \beta(k)=\left[1+\left(h^{\prime}-\Delta\right) e^{-i k}\right] e^{i(L+1) k} .
\end{aligned}
$$

The eigenvalues are given by

$$
E\left(\left\{k_{j}\right\}\right)=-\frac{1}{2}\left[(L-1) \Delta+h+h^{\prime}\right]+2 \sum_{j=0}^{M-1}\left(\Delta-\cos \left(k_{j}\right)\right) .
$$


The identity

$$
\frac{s\left(k^{\prime}, k\right)}{s\left(k, k^{\prime}\right)}=e^{i \Theta\left(k, k^{\prime}\right)}
$$

where $k, k^{\prime}, \Delta$ are real and $\Theta\left(k, k^{\prime}\right)$ is defined by

$$
\Theta\left(k, k^{\prime}\right)=2 \arctan \left[\frac{\Delta \sin \left(\frac{k-k^{\prime}}{2}\right)}{\Delta \cos \left(\frac{k-k^{\prime}}{2}\right)-\cos \left(\frac{k+k^{\prime}}{2}\right)}\right]
$$

implies that the ratio of $B$-functions appearing in the Bethe equations is given by

$$
\frac{B\left(-k, k^{\prime}\right)}{B\left(k, k^{\prime}\right)}=e^{i\left[\Theta\left(k, k^{\prime}\right)+\Theta\left(k,-k^{\prime}\right)\right]} .
$$

We observe the further identities

$$
\frac{\alpha(k)}{\alpha(-k)}=e^{i \Phi(k, h)}, \quad \frac{\beta(k)}{\beta(-k)}=e^{i \Phi\left(k, h^{\prime}\right)} e^{i 2(L+1) k},
$$

where $k, h$ are real and the function $\Phi(k, h)$ is defined by

$$
\Phi(k, h)=-2 \arctan \left[\frac{(h-\Delta) \sin (k)}{1+(h-\Delta) \cos (k)}\right] .
$$

Taking the logarithm of the Bethe equations (2.8) using (2.14) and (2.15), we obtain (assuming that all Bethe roots $k_{0}, \ldots, k_{M-1}$ are real)

$$
Z\left(k_{j} ;\left\{k_{l}\right\}\right)=2 \pi J_{j}, \quad j=0, \ldots, M-1,
$$

where $Z\left(k ;\left\{k_{l}\right\}\right)$ is the so-called counting function

$$
Z\left(k ;\left\{k_{l}\right\}\right)=2(L+1) k+\Phi(k, h)+\Phi\left(k, h^{\prime}\right)+\Theta(k,-k)-\sum_{l=0}^{M-1}\left[\Theta\left(k, k_{l}\right)+\Theta\left(k,-k_{l}\right)\right]
$$

and $J_{j}$ are distinct integers satisfying $\left\{J_{0}, \ldots, J_{M-1}\right\} \subset\{1, \ldots, L\}$. In contrast with the corresponding closed chain, the counting function (2.18) involves both $\Theta\left(k, k_{l}\right)$ and $\Theta\left(k,-k_{l}\right)$, and includes terms that depend on the boundary magnetic fields $h$ and $h^{\prime}$.

In our algorithm for constructing the Bethe states on a quantum computer, we compute the Bethe roots classically by solving (2.17) numerically by iteration. Namely, starting from $k_{j}^{(0)}=J_{j}$, we solve

$$
Z\left(k_{j}^{(n+1)} ;\left\{k_{l}^{(n)}\right\}\right)=2 \pi J_{j}, \quad j=0, \ldots, M-1, \quad n=0,1, \ldots,
$$

which converges rapidly. In this work we consider examples with $M \leq\left\lfloor\frac{L}{2}\right\rfloor$. 
We observe that the coefficients (2.6) satisfy

$$
\frac{A\left(k_{0}, \ldots, k_{j}, k_{l}, \ldots, k_{M-1}\right)}{A\left(k_{0}, \ldots, k_{l}, k_{j}, \ldots, k_{M-1}\right)}=e^{i \Theta\left(k_{j}, k_{l}\right)},
$$

as well as

$$
\frac{A\left(k_{0}, \ldots,-k_{j}, \ldots, k_{M-1}\right)}{A\left(k_{0}, \ldots, k_{j}, \ldots, k_{M-1}\right)}=e^{i k_{j}(2 L+2)} e^{i \Phi\left(k_{j}, h\right)} V\left(k_{j} ; k_{j+1}, \ldots, k_{M-1}\right),
$$

where

$$
V\left(k_{j} ; k_{j+1}, \ldots, k_{M-1}\right)=e^{i \sum_{l=j+1}^{M-1}\left[\Theta\left(-k_{j}, k_{l}\right)+\Theta\left(k_{l}, k_{j}\right)\right]} .
$$

While the relation 2.20 is true also for the corresponding closed chain, the relation (2.21) is a new feature of the open chain. Our algorithm for constructing Bethe states is based on the relations 2.20$)-(2.22$.

\section{Algorithm}

Our algorithm for preparing the $L$-qubit Bethe state 2.2 requires a minimum of $L+M^{2}+2 M$ qubits (hence, $M^{2}+2 M$ ancillas), which are allocated as follows:

- $L$ "system" qubits (designated by $s$ ), whose state becomes the Bethe state (2.2) on successful completion of the algorithm

- $M(M+1)$ "permutation-label" qubits (designated by $p$ ), which are used to implement the sum over all possible permutations and negations in the wave function (2.4), and to apply the phases in 2.20$)-(2.22)$ and the signs $\varepsilon_{P}$ in $(2.4)$

- $M$ "faucet" qubits (designated by $f$ ), which are used to apply the phase $e^{i \sum_{j=0}^{M-1} k_{j} x_{j}}$ in the wave function 2.4 .

In our implementation of the algorithm, we make use of two additional "work" qubits, in order to reduce the gate count.

The algorithm consists of the steps outlined in Algorithm 1, and which are described in more detail below.

Step 1 As already discussed in Sec. 2, given real values of the parameters $\Delta, h$ and $h^{\prime}$ and a set of $M$ distinct integers $\left\{J_{0}, \ldots, J_{M-1}\right\} \subset\{1, \ldots, L\}$, the first step of the algorithm is to determine the corresponding set of real Bethe roots $\left\{k_{0}, \ldots, k_{M-1}\right\}$ by classically solving 2.19). The corresponding energy eigenvalue is given by (2.11). 


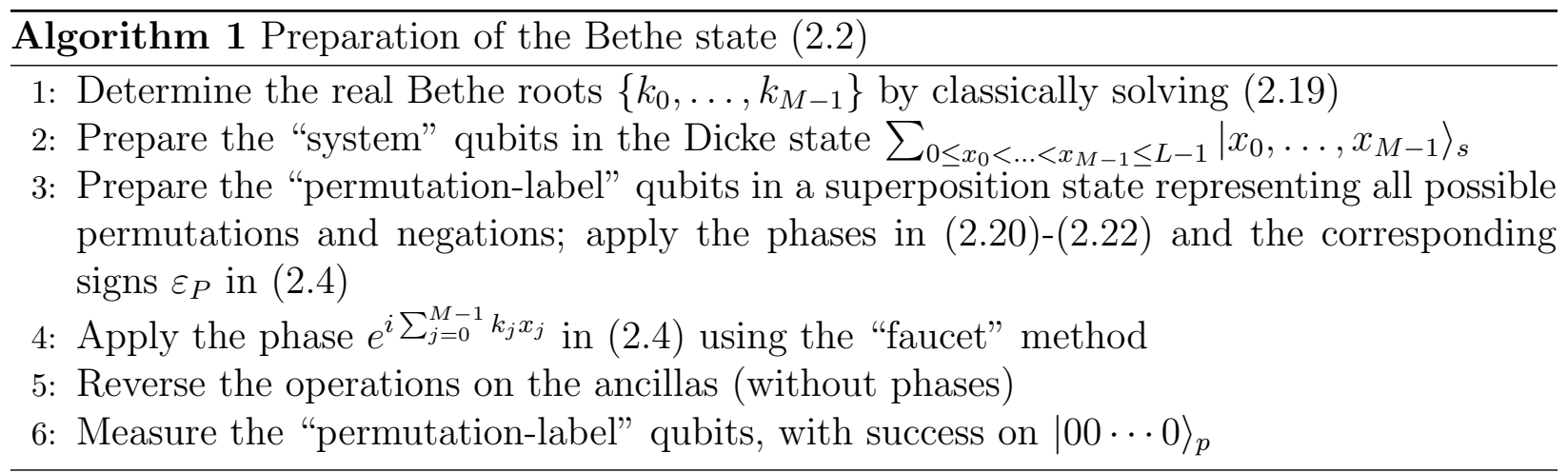

Step 2 This step of the algorithm is to prepare the "system" qubits in the so-called Dicke state

$$
\left|D_{L, M}\right\rangle_{s}=\sum_{0 \leq x_{0}<\ldots<x_{M-1} \leq L-1}\left|x_{0}, \ldots, x_{M-1}\right\rangle_{s}
$$

which is the same as the Bethe state (2.2) but with all the coefficients $f\left(x_{0}, \ldots, x_{M-1}\right)$ set to 1 . This state is an equal-weight superposition of the $\left(\begin{array}{l}L \\ M\end{array}\right) L$-qubit states with $M$ 1's and $L-M$ 0's. For example,

$$
\left|D_{3,1}\right\rangle=\frac{1}{\sqrt{3}}(|001\rangle+|010\rangle+|100\rangle) .
$$

A deterministic algorithm for constructing this state is already available [26], see [27] for an improved gate count.

Step 3 The goal of this step of the algorithm is to prepare the "permutation-label" qubits in the state

$$
\frac{1}{\sqrt{2^{M} M !}} \sum_{P} \varepsilon_{P} A\left(k_{0}, \ldots, k_{M-1}\right)|P\rangle_{p}
$$

As in [10], the phases $\varepsilon_{P} A\left(k_{0}, \ldots, k_{M-1}\right)$ are kicked back onto the "system" qubits, while $|P\rangle_{p}$ is used in step 4 to apply controlled gates. However, here $|P\rangle_{p}$ represents not only permutations, but also negations. The "permutation-label" register consists of $M$ subregisters, each of which consists of $M+1$ qubits, for a total of $M(M+1)$ qubits. In each subregister, the first $M$ qubits in the subregister, which we call the "hot" qubits, store an integer in the set $\{0, \ldots, M-1\}$ by means of "one-hot encoding". As an example, for the case $M=3$, the possible states of the "hot" qubits are $|0\rangle \equiv|001\rangle,|1\rangle \equiv|010\rangle,|2\rangle \equiv|100\rangle$. These integers serve to label the $M$ down spins in a given eigenstate and are used to efficiently generate the phases arising from the permutations that appear in (2.4). The last qubit in each subregister is a "reflection" qubit, whose state indicates the presence (when $|1\rangle$ ) or absence (when $|0\rangle$ ) of a negation associated with the given down spin in the corresponding term of (2.4).

To obtain an efficient method for state preparation of (2.2), we first implement the phases associated with negations on the various down spins, while the permutation part is trivial 
(i.e. represents only the identity permutation). We then execute the permutations and corresponding phase gates on the permutation-label, to fully produce all terms in (2.2). Starting with the "permutation-label" qubits in the initial state $|00 \ldots\rangle_{p}$, we encode the identity permutation by storing the values $0, \ldots, M-1$ in ascending order (from right to left). We next apply Hadamard gates to the reflection qubit in each sublabel to generate the superposition of having reflected/not reflected each down spin label. Again for the case $M=3$, the "permutation-label" qubits are in the state

$$
(H|0\rangle|100\rangle)(H|0\rangle|010\rangle)(H|0\rangle|001\rangle)
$$

We next apply the phases in 2.21$)$. The factor $e^{i k_{j}(2 L+2)} e^{i \Phi\left(k_{j}, h\right)}$ is simply implemented by applying to the $j^{\text {th }}$ "reflection" qubit a phase gate with angle $k_{j}(2 L+2)+\Phi\left(k_{j}, h\right)+$ $\pi$; the additional phase $\pi$ implements the corresponding sign $\varepsilon_{P}$ in (2.4). The factor $V\left(k_{j} ; k_{j+1}, \ldots, k_{M-1}\right)$ is implemented via an iterative process. Working backwards from down spin $j=M-2$ to $j=0$, the exponential factors for $l>j$ in 2.22 can be implemented using a phase gate acting on the reflection qubit of spin $j$. In principle, one ought to control on the state of the reflection qubit of spin $l$, since $k_{l}$ could be negative. However, owing to the identity $\Theta\left(-k_{j}, k_{l}\right)=\Theta\left(-k_{l}, k_{j}\right)$ for real $k_{i}$ and $\Delta$, one has that

$$
\Theta\left(-k_{j},-k_{l}\right)+\Theta\left(-k_{l}, k_{j}\right)=\Theta\left(-k_{j}, k_{l}\right)+\Theta\left(k_{l}, k_{j}\right)
$$

and so the factor ultimately appearing in $(2.22)$ is the same regardless of the sign of $k_{l}$. Thus, the summation in 2.22 can be performed classically and a single phase gate can be used to produce $V\left(k_{j} ; k_{j+1}, \ldots, k_{M-1}\right)$ for fixed $j$. In fact, the corresponding phase can be added to others appearing in (2.21), so that all these can be implemented simultaneously (along with the $\left.\operatorname{sign} \varepsilon_{P}\right)$.

Starting from the state representing the identity permutation such as (3.1), we now generate a state representing a superposition of the $M$ ! permutations by iteratively swapping subregisters, as explained in [10]. To implement the phase in $(2.20)$, we apply phase gates with angles $\Theta\left(\epsilon_{j} k_{j}, \epsilon_{l} k_{l}\right)+\pi$ after the subregisters $j$ and $l$ have been swapped; these gates are controlled in part by "reflection qubits" to ensure the proper signs $\epsilon= \pm 1$. The additional phase $\pi$ implements the corresponding sign $\varepsilon_{P}$ in $(2.4)$.

Step 4 This step of the algorithm is to apply the phase $e^{i \sum_{j=0}^{M-1} k_{j} x_{j}}$ in 2.4 . We use the faucet method [10], which exploits the fact that each $x_{j}$ is an integer; hence, the phase $e^{i \epsilon_{j} k_{j} x_{j}}$ can be applied by performing $x_{j}$ repeated applications of the phase $e^{i \epsilon_{j} k_{j}}$. All $M$ "faucet" qubits are initially turned on (i.e., placed in the state $|1\rangle$ ). We then loop through the $L$ "system" qubits. At each step, we check to see if the current system qubit represents a down spin (is in the state $|1\rangle$ ). If so, the next faucet qubit is turned off (set to $|0\rangle$ ), as the complete phase $e^{i \epsilon_{j} k_{j} x_{j}}$ for the given $k_{j}$ value has been produced. Moreover, as we loop through the "system" qubits, we also apply controlled phase gates with angle $\epsilon_{j} k_{j}$ on the "hot" qubits that are controlled by the $j^{\text {th }}$ "faucet" qubit (must be on), and by the $j^{\text {th }}$ "reflection" qubit (determines the sign $\epsilon_{j}$ ). 
Step 5 This step of the algorithm is to reverse the operations on the ancillas, except without applying any phases. After this step, the "faucet" qubits (but not necessarily all the "permutation-label" qubits) are in the $|0\rangle$ state; and the state $|\Psi\rangle$ of the quantum computer is given by

$$
|\Psi\rangle=\alpha|00 \ldots 0\rangle_{p}|00 \ldots 0\rangle_{f}|M\rangle_{s}+\ldots,
$$

where $|M\rangle_{s}$ is the normalized Bethe state (2.2), $\alpha$ is some complex number, and the ellipsis denotes additional terms that are orthogonal to the first one.

Step 6 The final step of the algorithm is to measure the "permutation-label" qubits. It follows from (3.3) that the "system" qubits are in the target normalized Bethe state $|M\rangle_{s}$ on the outcome $|00 \ldots 0\rangle_{p}$, with success probability $|\alpha|^{2}$.

\section{Simulations}

We have implemented Algorithm 1 using Qiskit, and we have executed the quantum circuit on the Statevector Simulator for values of $M$ up to $M=3$, and for values of $L$ up to $L=8$. We have verified that the state obtained by projecting the final state (3.3) to the subspace with all ancillas in the state $|0\rangle$ is indeed an exact eigenstate of the Hamiltonian (1.1), with eigenvalue (2.11).

Figure 1 shows the success probability $|\alpha|^{2}$ of the algorithm as a function of eigenstate energy for $M=2,3$ down spins and $L=6$ sites. As in the closed chain, there is a clear decrease of success probability with $M$, as well as with energy. Figure 2 illustrates the success probability for fixed $M=2$ and varying $L=4,5,6$. This also agrees with findings from the closed chain, in that the minimum success probability does not vary strongly with $L$. This suggests that at reasonably small $M$ one can maintain a high success probability for large $L$ systems, beyond what is classically tractable with numerical methods. Furthermore, amplitude amplification can be straightforwardly applied to enhance the success probability for the open chain, as was demonstrated explicitly for the closed case [10].

\section{Discussion}

We have formulated a probabilistic algorithm for preparing exact eigenstates of the Hamiltonian (1.1). Due to the significant depth of the circuit, its implementation on existing and near-term hardware may not be feasible. However, we expect that a fault-tolerant quantum computer with a few hundred qubits will outperform any classical computer on this task, given that the algorithm requires only $L+M^{2}+2 M$ qubits, while the Bethe eigenstate (2.2) is given by a sum of $\left(\begin{array}{c}L \\ M\end{array}\right) 2^{M} M$ ! terms. This algorithm is a generalization of the one for the corresponding closed chain [10], which requires $L+M^{2}+M$ qubits. The additional $M$ ancilla qubits in the open chain case are the reflection qubits that are used to keep track of the negations appearing in 2.4 . 


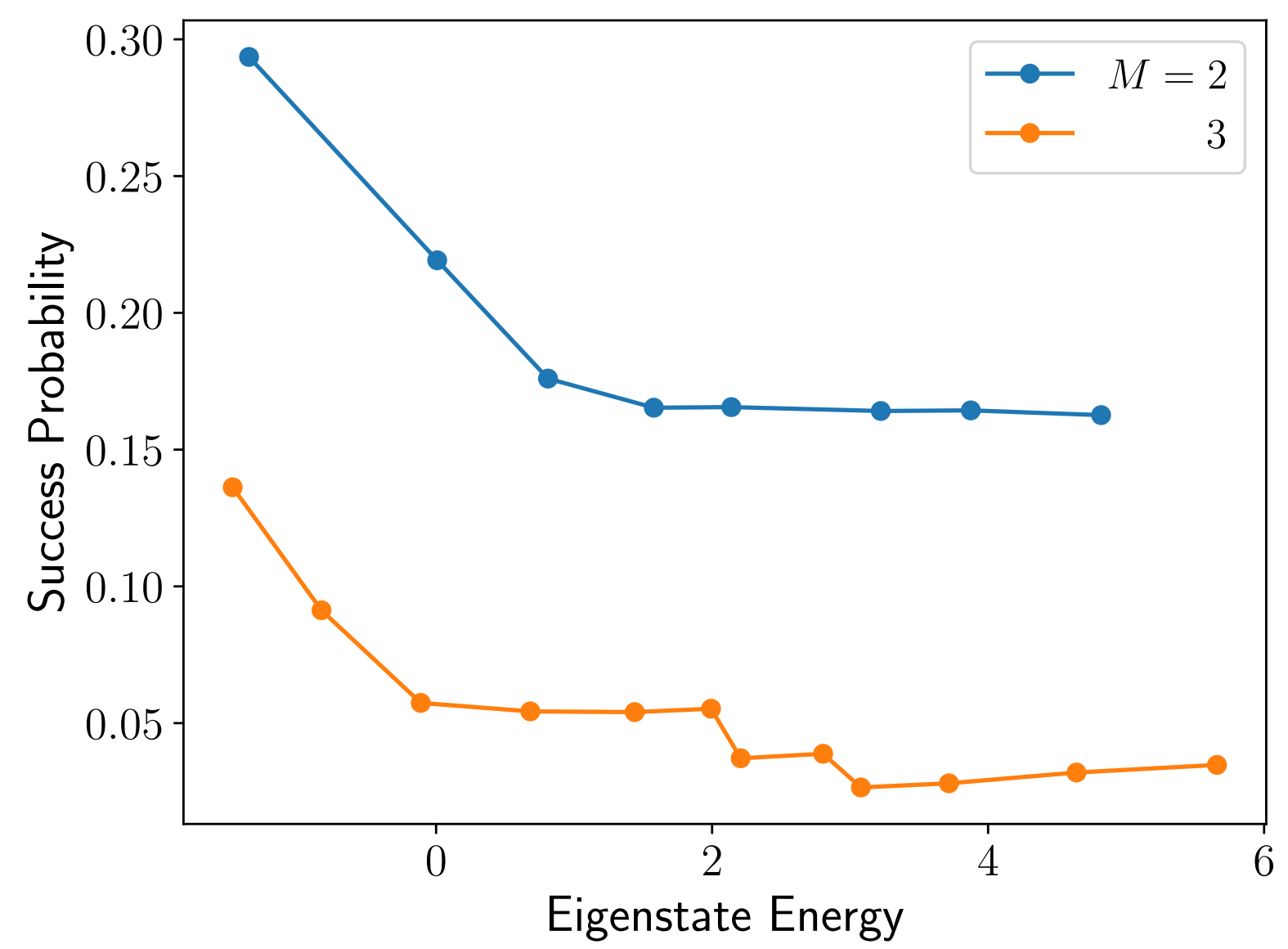

Figure 1: Success probability of the open chain Bethe ansatz state preparation algorithm as a function of eigenstate energy, for $M=2,3$ and $L=6$. Other parameters are $\Delta=0.5$, $h=0.1, h^{\prime}=0.3$. 


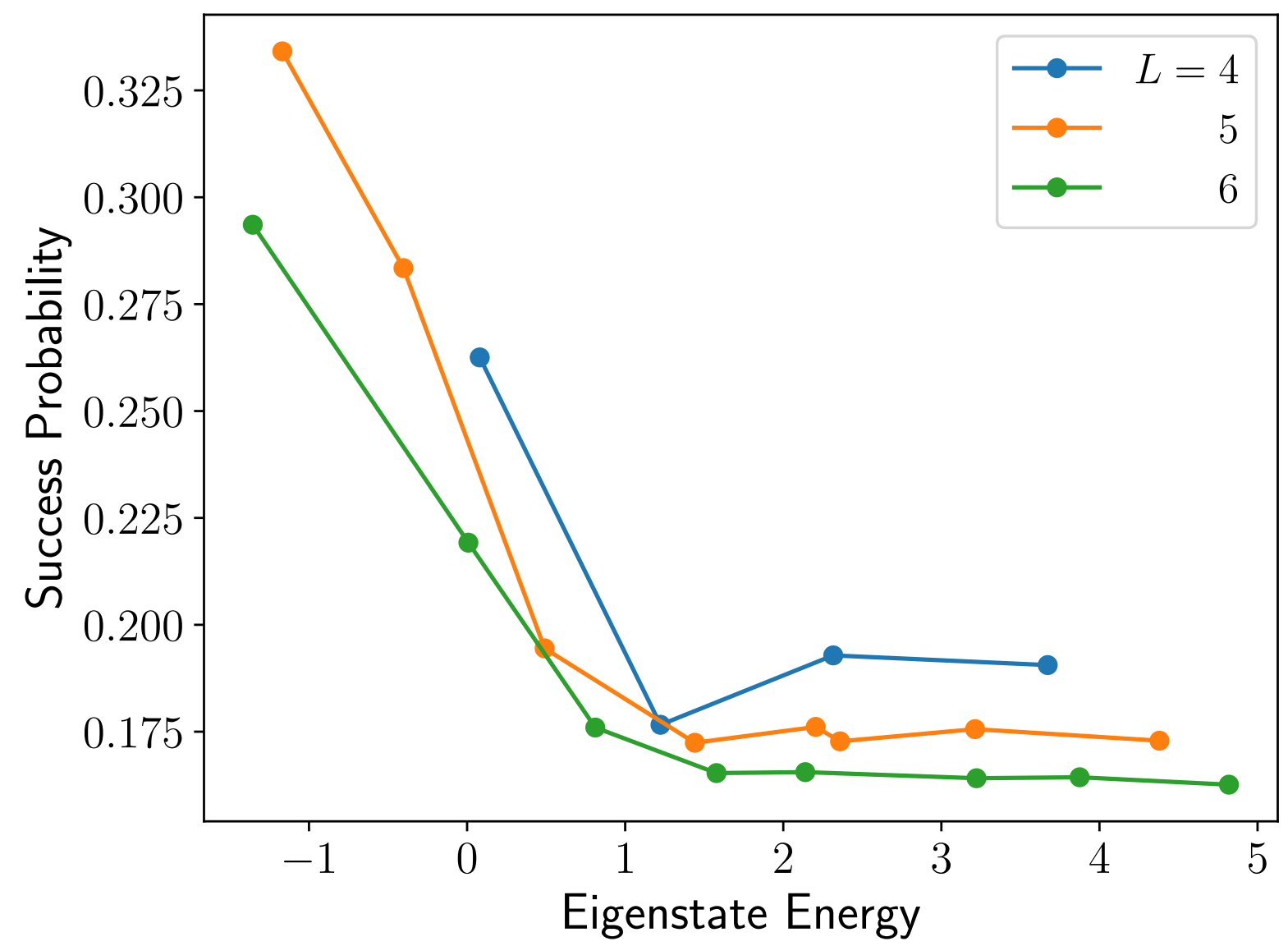

Figure 2: Success probability of the open chain Bethe ansatz state preparation algorithm as a function of eigenstate energy, for $L=4,5,6$ and $M=2$. Other parameters are $\Delta=0.5$, $h=0.1, h^{\prime}=0.3$. 
We expect that it will be possible to use the eigenstates furnished by this algorithm to explicitly compute ground-state correlation functions. Indeed, at least for some range of parameter values, the ground state of the antiferromagnetic Hamiltonian (i.e., $-\mathcal{H}$, with $\mathcal{H}$ given by (1.1) ) is described by $M=L / 2$ real Bethe roots, and can therefore be obtained using this algorithm. Moreover, standard routines exist for evaluating expectation values of products of Pauli operators on a quantum computer.

Of course, not all eigenstates of this Hamiltonian can be described by real Bethe roots. It remains an interesting challenge to prepare generic eigenstates, which would require solving two main problems: (1) finding an efficient algorithm for determining the (complex) Bethe roots; and (2) generalizing the state-preparation algorithm to the case of complex Bethe roots. Indeed, for the former problem, neither a classical nor a quantum algorithm is available; even for the simpler case of the closed XXX chain with periodic boundary conditions, the complete set of Bethe roots is known for at most $L=14$ sites [28]. Regarding the latter

problem: for complex Bethe roots, the factors in 2.20), 2.21 as well as $e^{i \sum_{j=0}^{M-1} k_{j} x_{j}}$ are no longer phases, and therefore those factors can no longer be simply implemented using controlled phase gates. Overcoming this problem would have the additional benefit of allowing the treatment of the $U_{q}(s u(2))$-invariant case $(1.2)$ with $q$ a root of unity. In that case the boundary magnetic fields $h, h^{\prime}$ are no longer real, and therefore the Hamiltonian (1.1) is no longer Hermitian. Nevertheless, its spectrum is real, and can be related to the spectra of $c<1$ minimal conformal field theories 19,22 .

We expect that the algorithm presented here for preparing Bethe states of the model (1.1) can be extended to other quantum integrable models with open boundary conditions that have been solved by coordinate Bethe ansatz (e.g. [29, 30]). It would also be interesting to formulate such algorithms that are based instead on algebraic Bethe ansätze, with which models have also been solved (see e.g. [20, 31, 34] and references therein). Although a deterministic approach for constructing algebraic Bethe ansatz states seems difficult [9], a probabilistic approach based on the linear combination of unitaries method [35, 36] - the strategy employed here and in [10] - may be more feasible.

\section{Acknowledgments}

E.B. acknowledges support from the Department of Energy, Award No. DE-SC0019199. S.E.E. acknowledges the DOE Office of Science, National Quantum Information Science Research Centers, Co-design Center for Quantum Advantage (C2QA), contract number DESC0012704. 
A Circuit for $L=4, M=2$
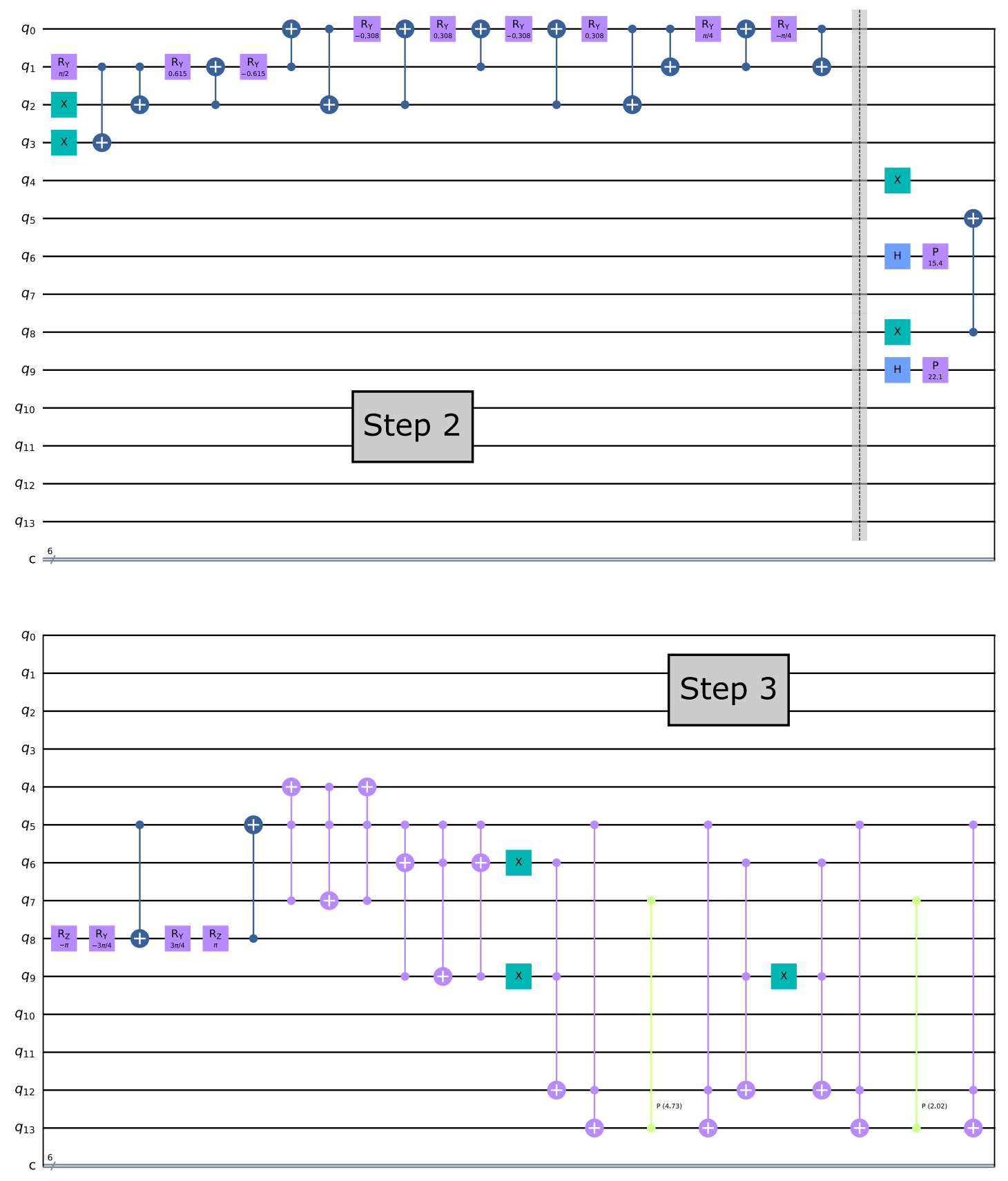

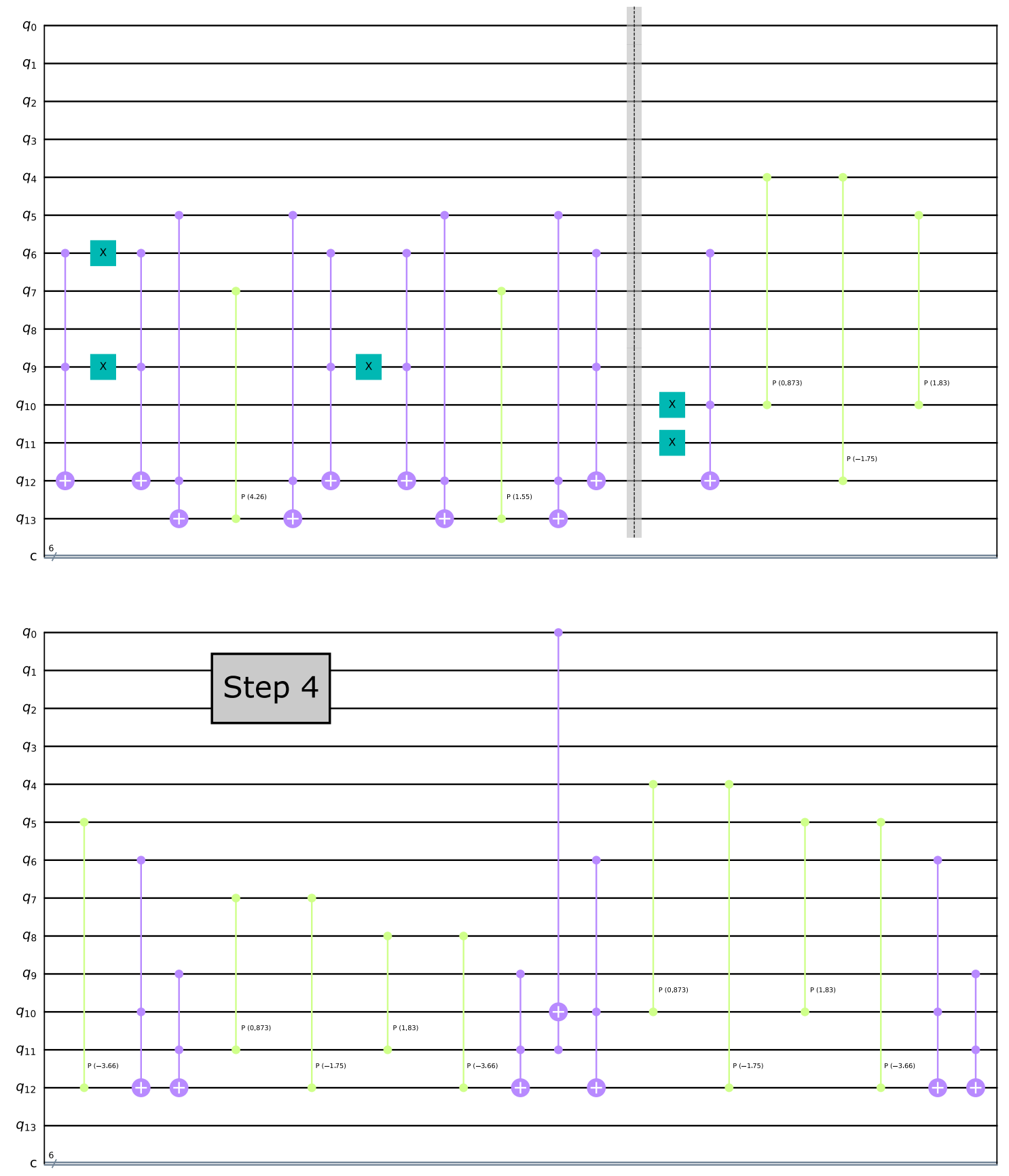

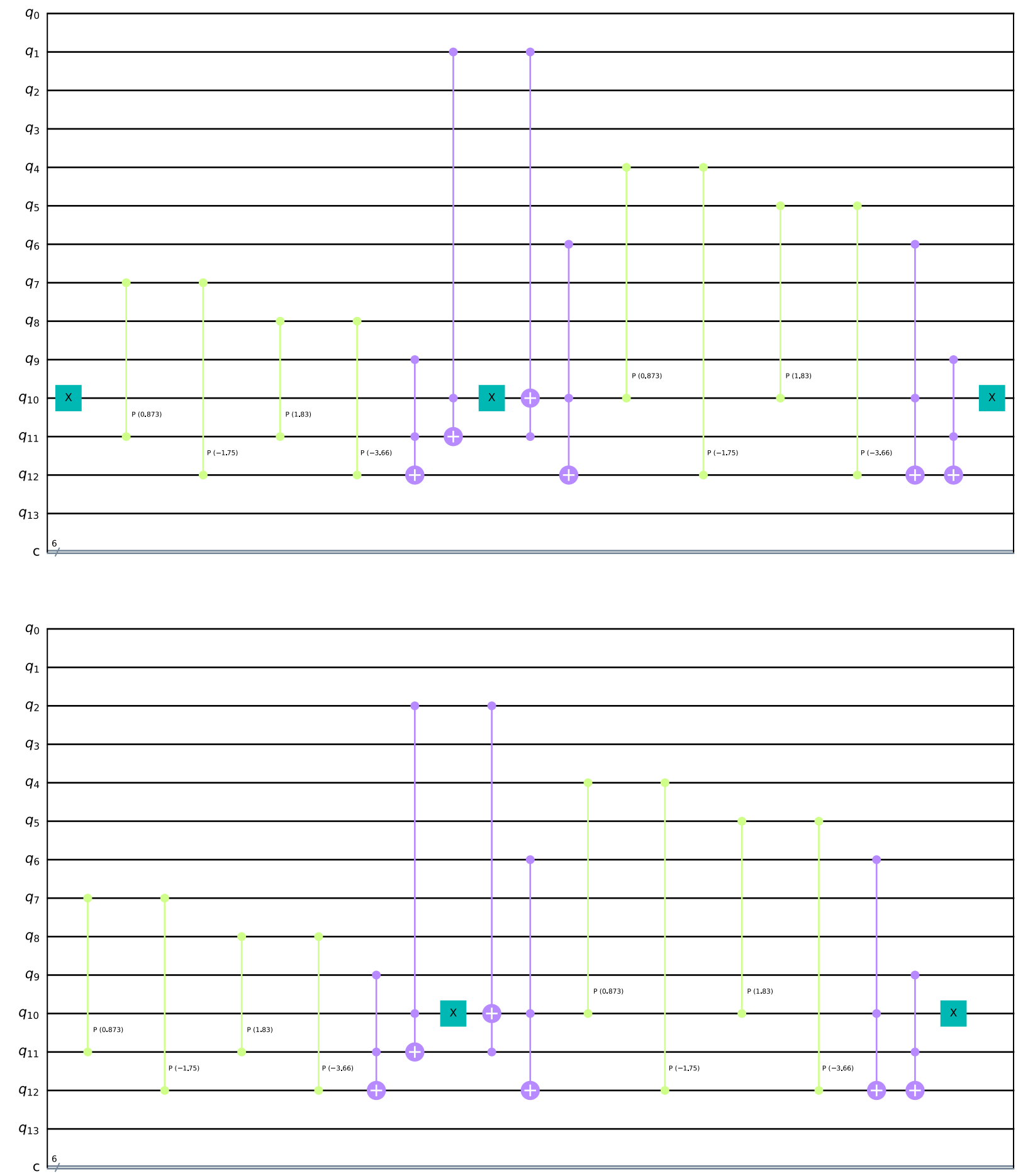

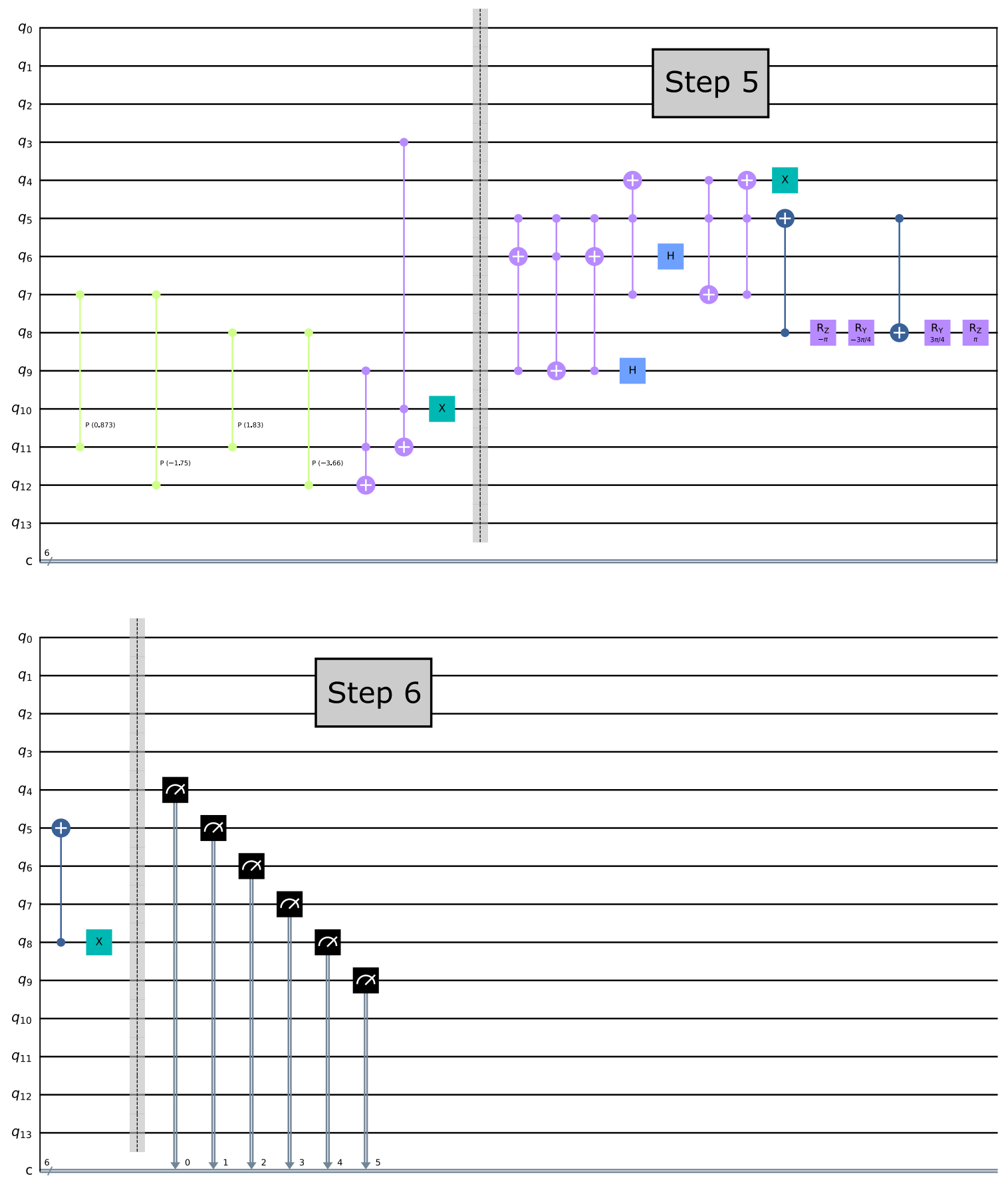

Figure 3: Quantum circuit to prepare an eigenstate of the open XXZ chain for $L=4$ and $M=2$. Other parameters are $\Delta=0.5, h=0.1, h^{\prime}=0.3$. The eigenstate is specified by the Bethe roots $k_{1}=0.8725655419522633$ and $k_{2}=1.8281634948690795$. The various steps of the algorithm, which are separated by barriers, are labeled; these steps are described in the corresponding parts of Sec. 3 . 


\section{References}

[1] H. Bethe, "On the theory of metals. 1. Eigenvalues and eigenfunctions for the linear atomic chain," Z. Phys. 71 (1931) 205-226.

[2] L. D. Faddeev, "How algebraic Bethe ansatz works for integrable models," in Symétries Quantiques (Les Houches Summer School Proceedings vol 64), A. Connes, K. Gawedzki, and J. Zinn-Justin, eds., pp. 149-219. North Holland, 1998. arXiv:hep-th/9605187 [hep-th].

[3] M. Jimbo, "Introduction to the Yang-Baxter Equation," Int. J. Mod. Phys. A 4 (1989) 3759-3777.

[4] M. T. Batchelor, "The Bethe ansatz after 75 years," Phys. Today 60 (2007) 36.

[5] M. A. Nielsen and I. L. Chuang, Quantum computation and quantum information. Cambridge University Press, 2019.

[6] N. D. Mermin, Quantum computer science, an introduction. Cambridge University Press, 2007.

[7] Y. Cao, J. Romero, J. P. Olson, M. Degroote, P. D. Johnson, M. Kieferová, I. D. Kivlichan, T. Menke, B. Peropadre, N. P. D. Sawaya, S. Sim, L. Veis, and A. Aspuru-Guzik, "Quantum Chemistry in the Age of Quantum Computing," Chemical Reviews 119 no. 19, (Aug., 2019) 10856-10915, arXiv:1812.09976 [quant-ph].

[8] S. McArdle, S. Endo, A. Aspuru-Guzik, S. C. Benjamin, and X. Yuan, "Quantum computational chemistry," Reviews of Modern Physics 92 no. 1, (Mar., 2020) 015003, arXiv:1808.10402 [quant-ph].

[9] R. I. Nepomechie, "Bethe ansatz on a quantum computer?," Quantum Information \&6 Computation 21 (2021) 255-265, arXiv:2010.01609 [quant-ph].

[10] J. S. Van Dyke, G. S. Barron, N. J. Mayhall, E. Barnes, and S. E. Economou, "Preparing Bethe Ansatz Eigenstates on a Quantum Computer," PRX Quantum 2 (2021) 040329, arXiv:2103.13388 [quant-ph].

[11] M. Cerezo, A. Arrasmith, R. Babbush, S. C. Benjamin, S. Endo, K. Fujii, J. R. McClean, K. Mitarai, X. Yuan, L. Cincio, and P. J. Coles, "Variational quantum algorithms," Nature Reviews Physics 3 no. 9, (Sept., 2021) 625-644, arXiv:2012.09265 [quant-ph].

[12] K. Bharti, A. Cervera-Lierta, T. H. Kyaw, T. Haug, S. Alperin-Lea, A. Anand, M. Degroote, H. Heimonen, J. S. Kottmann, T. Menke, W.-K. Mok, S. Sim, L.-C. Kwek, and A. Aspuru-Guzik, "Noisy intermediate-scale quantum (NISQ) algorithms,"

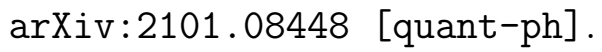


[13] D. Wecker, M. B. Hastings, and M. Troyer, "Progress towards practical quantum variational algorithms," Physical Review A 92 no. 4, (Oct., 2015) 042303, arXiv:1507.08969 [quant-ph].

[14] J. F. Gonthier, M. D. Radin, C. Buda, E. J. Doskocil, C. M. Abuan, and J. Romero, "Identifying challenges towards practical quantum advantage through resource estimation: the measurement roadblock in the variational quantum eigensolver," arXiv:2012.04001 [quant-ph].

[15] V. von Burg, G. H. Low, T. Häner, D. S. Steiger, M. Reiher, M. Roetteler, and M. Troyer, "Quantum computing enhanced computational catalysis," Physical Review Research 3 no. 3, (July, 2021) 033055, arXiv:2007.14460 [quant-ph].

[16] J. Preskill, "Quantum Computing in the NISQ era and beyond," Quantum 2 (Aug, 2018) 79, arXiv:1801.00862 [quant-ph].

[17] R. Orbach, "Linear Antiferromagnetic Chain with Anisotropic Coupling," Phys. Rev. 112 (1958) 309-316.

[18] M. Gaudin, "Boundary energy of a Bose gas in one dimension," Phys. Rev. A 4 (1971) 386-394.

[19] F. C. Alcaraz, M. N. Barber, M. T. Batchelor, R. J. Baxter, and G. R. W. Quispel, "Surface exponents of the quantum XXZ, Ashkin-Teller and Potts models," J. Phys. A20 (1987) 6397.

[20] E. K. Sklyanin, "Boundary conditions for integrable quantum systems," J. Phys. A21 (1988) 2375.

[21] S. Ghoshal and A. B. Zamolodchikov, "Boundary S matrix and boundary state in two-dimensional integrable quantum field theory," Int. J. Mod. Phys. A9 (1994) 3841-3886, arXiv:hep-th/9306002 [hep-th]. [Erratum: Int. J. Mod. Phys.A9,4353 (1994)].

[22] V. Pasquier and H. Saleur, "Common structures between finite systems and conformal field theories through quantum groups," Nucl. Phys. B330 (1990) 523-556.

[23] P. P. Kulish and E. K. Sklyanin, "The general $U_{q}(s l(2))$ invariant XXZ integrable quantum spin chain," J. Phys. A24 (1991) L435-L439.

[24] N. Kitanine, K. K. Kozlowski, J. M. Maillet, G. Niccoli, N. A. Slavnov, and V. Terras, "Correlation functions of the open XXZ chain I," J. Stat. Mech. 0710 (2007) P10009, arXiv:0707.1995 [hep-th].

[25] N. Kitanine, K. K. Kozlowski, J. M. Maillet, G. Niccoli, N. A. Slavnov, and V. Terras, "Correlation functions of the open XXZ chain II," J. Stat. Mech. 0807 (2008) P07010, arXiv:0803.3305 [hep-th]. 
[26] A. Bärtschi and S. Eidenbenz, "Deterministic preparation of Dicke states," Lecture Notes in Computer Science (2019) 126-139, arXiv:1904.07358 [quant-ph].

[27] C. S. Mukherjee, S. Maitra, V. Gaurav, and D. Roy, "On actual preparation of Dicke state on a quantum computer," IEEE Trans. Quant. Eng. 1 (2020) 3102517, arXiv:2007.01681 [quant-ph].

[28] W. Hao, R. I. Nepomechie, and A. J. Sommese, "Completeness of solutions of Bethe's equations," Phys. Rev. E 88 no. 5, (2013) 052113, arXiv:1308.4645 [math-ph].

[29] H. Asakawa and M. Suzuki, "Finite-size corrections in the XXZ model and the Hubbard model with boundary fields," Journal of Physics A Mathematical General 29 no. 2, (Jan., 1996) 225-245.

[30] E. Fireman, A. Lima-Santos, and W. Utiel, "Bethe ansatz solution for quantum spin-1 chains with boundary terms," Nuclear Physics B 626 no. 3, (Apr, 2002) 435-462, arXiv:0110048 [nlin].

[31] X.-W. Guan, "Algebraic Bethe ansatz for the one-dimensional Hubbard model with open boundaries," Journal of Physics A: Mathematical and General 33 no. 30, (Aug., 2000) 5391, arXiv:9908054 [cond-mat].

[32] G.-L. Li and K.-J. Shi, "The algebraic Bethe ansatz for open vertex models," Journal of Statistical Mechanics: Theory and Experiment 2007 no. 01, (Jan, 2007) P01018-P01018, arXiv:0611127 [hep-th].

[33] S. Belliard and E. Ragoucy, "The nested Bethe ansatz for "all" open spin chains with diagonal boundary conditions," Journal of Physics A: Mathematical and Theoretical 42 no. 20, (Apr, 2009) 205203, arXiv:0902.0321 [math-ph].

[34] A. Gerrard and V. Regelskis, "Nested algebraic Bethe ansatz for orthogonal and symplectic open spin chains," Nuclear Physics B 952 (Mar., 2020) 114909, arXiv:1909.12123 [math-ph].

[35] A. M. Childs and N. Wiebe, "Hamiltonian simulation using linear combinations of unitary operations," Quantum Information \& Computation 12 (November, 2012) 901, arXiv:1202.5822 [quant-ph].

[36] D. W. Berry, A. M. Childs, R. Cleve, R. Kothari, and R. D. Somma, "Simulating Hamiltonian Dynamics with a Truncated Taylor Series," Physical Review Letters 114 no. 9, (Mar., 2015) 090502, arXiv:1412.4687 [quant-ph]. 